\title{
A Power Saving Scheme for Heterogeneous Wireless Access Networks`
}

\author{
SuKyoung Lee, LaeYoung Kim, and Hojin Kim \\ Dept. of Computer Science, Yonsei University, Seoul, Korea \\ sklee@cs.yonsei.ac.kr
}

\begin{abstract}
In integrated WLAN and cellular networks, we propose a power saving scheme that completely turns off WLAN interface in the idle state and wakes it up when there is incoming data from long-lived traffic. We also develop a Mobile IPv6 (MIPv6)-based signaling to turn on the WLAN interface only for long-lived traffic. It is shown via simulations that the proposed power saving scheme improves power efficiency over typical WLAN system.
\end{abstract}

Keywords: heterogeneous networks, power saving, MIPv6.

\section{Introduction}

In the integrated Wireless LAN (WLAN) and cellular networks, power efficiency is still one of the most important aspects as in existing wireless networks. To keep the WLAN card operating, mobile nodes (MNs) with dual interfaces have to remain on, but in idle state. In [1, although the WLAN interface can be turned off in the idle state, MNs should still listen to the lower-power radio for the idle state. In [2], a power saving scheme is proposed to completely turn off the WLAN interface, nevertheless, this scheme cannot easily support third-party WLAN since it is based on tightly-coupled interworking architecture. Therefore, we propose a power saving scheme based on loosely-coupled interworking architecture to completely turn off WLAN interface of dual-mode MN and wake it up when there is a need to receive data. Further, a MIPv6-based signaling is developed to turn on the WLAN interface only for long-lived traffic so that the WLAN interface should not be turned on and off repeatedly for a momentary traffic, dissipating power. We show via simulations that the proposed power saving scheme improves power efficiency over existing WLAN.

\section{Idle Power Saving Scheme}

A typical WLAN interface has active and idle states. In idle state with powersave mode, power consumption level for the WLAN interface is several times as high as that of cellular interface [1]. In our scheme, to save the power in idle

\footnotetext{
* This work was supported by grant No.R01-2006-000-10614-0 from the Basic Research Program of the Korea Science \& Engineering Foundation.
} 


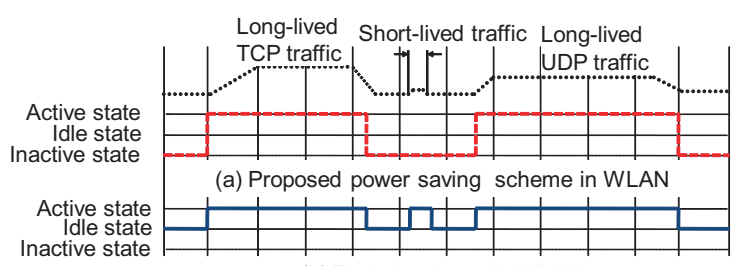

(b) Typical scheme in WLAN

Fig. 1. Idle power saving for WLAN interface

state (so called "idle power"), WLAN interface is turned off without any periodic wake-up during the idle state, that is called "inactive" state in this paper and is woke up when there is incoming data from long-lived traffic depending on the duration and amount of data (e.g., file transfer, streaming service). Once the WLAN interface is turned on, it enters active state to receive the data and then returns to inactive state after the data session is completed as shown in Fig. 1] In this study, we focus on downlink traffic since it is envisioned that $4^{\text {th }}$ generation wireless system's traffic pattern will be highly asymmetrical. Our proposed scheme targets on the loosely-coupled interworking (based on MIP) the cost of which is lower compared to the tightly-coupled interworking because WLAN is deployed as an independent operator connected to the Internet.

In our system model, the cellular interface is assumed to be able to detect Access Points (APs) by listening to paging channel. Basing our system on 3GPP system, Gateway GPRS Support Node (GGSN) is responsible for controlling IP connections between users and external data networks (i.e. Internet) with its own buffer. To reduce the time taken to wake up the WLAN interface, each AP is configured with a special Service Set Identifier (SSID) string which provides prefix information of the attached 802.11 gateway as in [3], resulting in fast configuration of MN's new Care-of-Address (CoA). The SSID field in beacon message consists of prefix of WLAN gateway's IP address, slash, prefix-length, one blank and identity of service set (e.g., 2001:1302:A83B:1104/64 AP1). As shown in Fig. 2, the developed signaling procedures proceed as follows :

1. When a Correspondent Node $(\mathrm{CN})$ has a long-lived traffic, it sets the flow label field in the IP packets to one (i.e., 0x00001) and two (i.e., 0x00002) for UDP and TCP traffics, respectively.

2. When the GGSN receives the packet whose flow label field is not zero, it sends a WAKEUP_WLAN message to the MN. This message is introduced as a new MIPv6 message whose Mobility Header (MH) type is 10 . The GGSN keeps all the TCP packets of loss-sensitive application, destined to the $\mathrm{MN}$ in its buffer to prevent the packet loss while switching to the WLAN interface. On the other hand, the GGSN continuously sends the UDP packets of delay-sensitive application to the MN.

3. On receiving the WAKEUP_WLAN message, the MN turns on its WLAN interface and is associated with an appropriate AP by performing beacon 


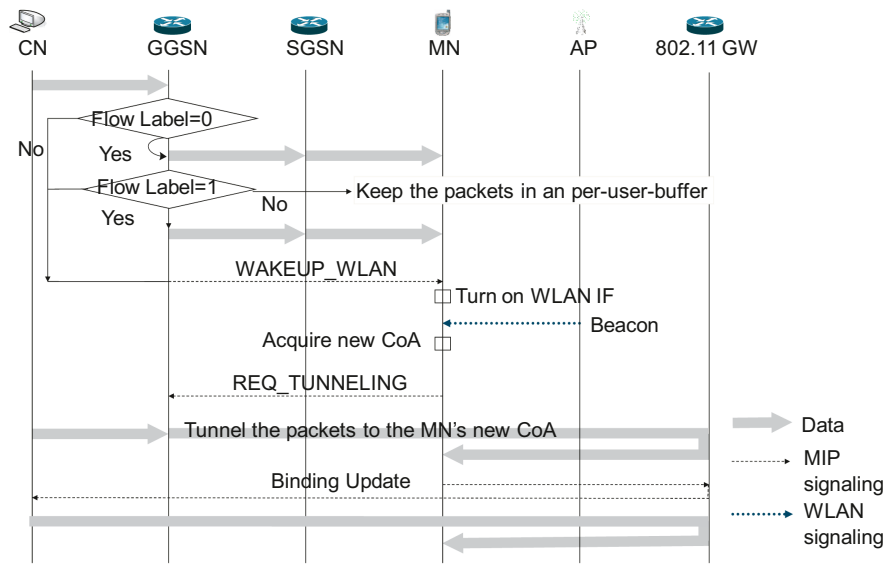

Fig. 2. MIPv6-based signaling procedures for incoming data traffic

scanning. Then, the MN configures a new CoA based on the prefix of the gateway's IP address obtained from the SSID field in the beacon message.

4. Once the new CoA is configured, the MN sends an REQ_TUNNELING message containing the new CoA, to the GGSN. This message is carried in a new MIPv6 message whose MH type is 11 . The MN also sends a Binding Update (BU) message to inform the $\mathrm{CN}$ and its home agent of the new CoA.

5. On receiving the REQ_TUNNELING message, the GGSN tunnels on-the-fly packets as well as the buffered packets (if there is any) to the new CoA.

6. When the $\mathrm{CN}$ receives the $\mathrm{BU}$, it sends the packets to the MN's new CoA indicated in the BU.

\section{Performance Evaluation}

Simulation experiments are carried to evaluate the performance of the proposed scheme by using ns- 2 with wireless extensions. The data session holding time is exponentially distributed with means of $5 \mathrm{~min}$ and $1 \mathrm{~min}$ for long-lived and shortlived traffics, respectively. Fig. 3 shows the network topology and the powers (W)

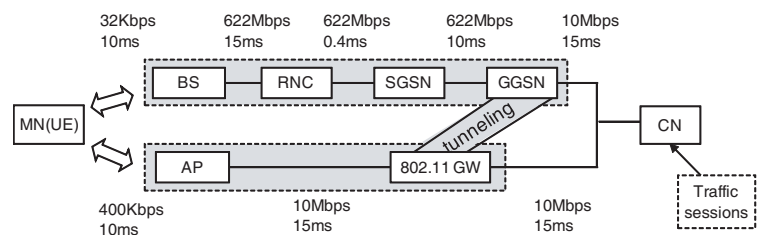

(a) Network Topology

\begin{tabular}{c|c|c}
\hline Interface & State & Power(W) \\
\hline \multirow{2}{*}{ WLAN } & active & 1.65 \\
\cline { 2 - 3 } & idle & 1.15 \\
\hline Cellular & active & 1.254 \\
\cline { 2 - 3 } & idle & 0.125 \\
\hline
\end{tabular}

(b) Power Consumption

Fig. 3. Network topology and power consumption parameters 


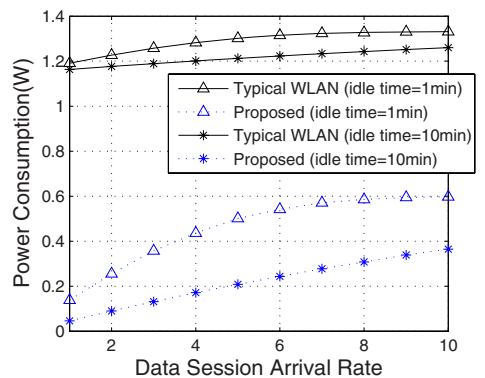

(a) When average idle time is $1 \mathrm{~min}$ and $10 \mathrm{~min}$

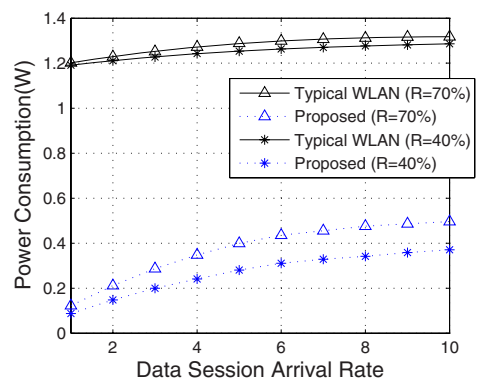

(b) When $\mathrm{R}$ is $70 \%$ and $40 \%$

Fig. 4. Total power consumption versus data session arrival rate

consumed by WLAN and cellular network interfaces in active and idle modes, which are obtained from [1] and 4].

Fig. 4 (a) shows the total power consumption (W) versus data session arrival rate when the average idle time is $1 \mathrm{~min}$ and $10 \mathrm{~min}$. The power consumption for our scheme is less than the typical WLAN over all the ranges of data session arrival rate. The performance improvement by our scheme is higher when the average idle time is $10 \mathrm{~min}$ than when $1 \mathrm{~min}$. Fig. 4 (b) shows the total power consumption versus data session arrival rate for the portion of long-lived traffic among total data sessions, $R=70 \%$ and $40 \%$. The proposed scheme achieves better power efficiency than the typical WLAN for the two values of $R$. However, the average improvement by the proposed scheme over the typical WLAN for $R=70 \%$ is smaller than that for $R=40 \%$ because the larger the portion of long-lived traffic is, the longer our scheme has to keep the WLAN interface on.

\section{Conclusion}

The simulation results showed that the proposed power saving scheme outperforms typical WLAN with respect to power saving by completely turning off the WLAN interface of dual-mode MN for the idle state and waking it up for long-lived traffic.

\section{References}

1. Shih, E., Bahl, P., Sinclair, M.: Wake on Wireless: An Event Driven Energy Saving Strategy for Battery Operated Devices. Mobicom, (Sep. 2002) 160-171.

2. Lee, S., Seo, S., Golmie, N.: An Efficient Power-Saving Mechanism for Integration of WLAN and Cellular Networks. IEEE Commun.Letters, Vol.9, No.12 (Dec. 2005) 1052-1054.

3. Jordan, N., Poropatich, A., Fleck, R.: Link-layer Support for Fast Mobile IPv6 Handover in Wireless LAN based Networks. IEEE LANMAN, (Apr. 2004) 139-143.

4. Baiamonte, V., Chiasserini, C.: Investigating MAC-layer Schemes to Promote Doze Mode in 802.11-based WLANs. IEEE VTC, Vol.3 (Oct. 2003) 1568-1572. 